\title{
"TEACHERS FLIPPING OUT" BEYOND THE ONLINE LECTURE: MAXIMISING THE EDUCATIONAL POTENTIAL OF VIDEO
}

\author{
Andrew Thomson \\ Creative Industries Faculty \\ Queensland University of Technology, Australia \\ as.thomson@qut.edu.au \\ Ruth Bridgstock \\ Creative Industries Faculty \\ Queensland University of Technology, Australia \\ r.bridgstock@qut.edu.au \\ Christiaan Willems \\ Creative Industries Faculty \\ Queensland University of Technology, Australia \\ c.willems@qut.edu.au
}

\begin{abstract}
While video is recognised as an important medium for teaching and learning in the digital age, many video resources are not as effective as they might be, because they do not adequately exploit the strengths of the medium. Presented here are some case studies of video learning resources produced for various courses in a university environment. This ongoing project attempts to identify pedagogic strategies for the use of video; learning situations in which video has the most efficacy; and what production techniques can be employed to make effective video learning resources.
\end{abstract}

Keywords

online learning, higher education, pedagogic strategy, video

\section{Introduction}

Online learning is now ubiquitous in higher education. Audiovisual material, including video, is an integral element of online learning. As the digital infrastructure of universities improves and student access to computers and data-enabled mobile devices increases, powerful learning possibilities are opened up. Students can potentially download and stream media wherever and whenever they like, affording great flexibility in learning experiences, and potentially de-tethering learning from the bricks and mortar university classroom. 
Aside from the great advantage of flexibility, video can be a very powerful teaching medium. Effective use of media engages students, aids depth and retention of knowledge, motivates interest in learning, and can be used to illustrate relevance of concepts (Mateer, 2011). While there is a wealth of literature providing support and advice on how to develop and teach online and blended courses generally, there has been relatively little consideration of (a) which pedagogic strategies can be employed to make the most of video content, (b) in which learning situations should video be used (rather than other learning resources), and (c) what constitutes an effective video for learning and teaching. These are important issues for university teachers to consider particularly given that making and uploading videos can be, compared with traditional lecture preparation, a time and resource-intensive exercise.

In addressing the above questions, this position paper shares the experiences of the authors in developing and teaching with video over a one year period in a large Creative Industries Faculty in a University of Technology in Australia. We note here that there is also much educational potential in working with students to make videos (Keegan, 2010; Kiili, Kauppinen, \& Laurinen, 2013) but our emphasis in this paper is on developing the capabilities of academic staff to produce and use video effectively in educational contexts about which there has been less published to date. Two of the authors of this paper are members of a small media unit that works with faculty to build video content while the third is a teaching staff member who uses video in large core undergraduate courses of $>700$ enrolments.

\section{Video for teaching in higher education: Current practices}

The university we work for is an Australian University of Technology. It is so named because it emphasises applied "real world" research and teaching in its practices. Within this university, we work for a large Creative Industries Faculty which confers undergraduate and postgraduate degrees in a wide range of media, entertainment, creative arts, and design disciplines. One of the disciplines in the Faculty is Film, Television and New Media, and, as the result of this, we have access to a state of the art multi-cam studio including green screen, multiple professional-level edit suites and numerous items of video production equipment. Despite our university's focus on technology and application and the fact that our Faculty has both significant media production teaching expertise and facilities for production of media, when we started with our project, there were very few teachers who were making their own videos for teaching or sourcing videos from elsewhere to use in teaching.

Our small video unit was set up as a less resource-intensive, more responsive and agile alternative to the traditional media production units used by universities that produce broadcast quality multiple-camera or studio-based video resources. For us, emphasis is on "fit-for-purpose quality," and content is usually filmed with one camera and one radio lapel microphone, using available light only. The teacher and production team collaborate on scripting and storyboarding, with postproduction undertaken by a video editor (often the same person who operated the camera) using a desktop computer running Adobe Premiere. Additional image or video “overlay" material is supplied by the teacher or sourced/created by the production team. Each member of the production team possesses multiple skill sets and, within one production, may need to serve as producer, director, cinematographer, sound recordist, animator, editor, and to some extent, learning designer.

When we commenced our project, the teaching practices in our Faculty were very similar to those commonly documented as going on in universities elsewhere in the world at present (Hogue, 2013). "Web lecture" variants have become part of the teaching repertoire largely because of a centrally-mandated lecture “capture system” that records a teacher's voice as he/she teaches in a live classroom. The capture system then pairs the voice with the teacher's slides and makes these available for student download on demand via the learning management system. A few teachers had also been experimenting with "flipping the classroom” (Bishop \& Verleger, 2013), in which asynchronous video lectures, often webcam-filmed in the teacher's office, were placed online to be viewed by students prior to attending weekly tutorials or workshops. 
Both of these uses of video can be argued to be "transmissive" approaches to teaching in that they are direct instruction methods derived from behaviourist principles. Transmissive approaches to teaching remain among the most common in universities today because they are relatively straightforward and efficient. Preparation time for a lecture is much less than for other approaches, a large number of students can be reached at once in a lecture hall, and universities are consistently set up, both in terms of infrastructure and entrenched culture/history, to teach in these ways. The lecture capture and "flipped classroom" lectures have, however, an added advantage -- the student is able to download, watch, and re-watch the lecture on demand.

\section{Which pedagogic strategies make the most of video content?}

Systematic study into learning processes in online and blended learning consistently suggests that active, problem-based learning activities premised upon a constructivist ideology are preferred over lecture approaches for depth of learning, meaning making, and application of knowledge (Hakkarainen, Saarelainen, \& Ruokamo, 2007). However, the lecture (including the e-lecture) remains entrenched in traditional teaching practice for the previously cited reasons and also, arguably, because of habit - the vast majority of academic teachers were educated in a transmissive paradigm and have themselves adopted lecture approaches for their entire working lives. As Demetriadis and Pombortsis (2007) stated, online lectures “increase flexibility of learning ... but only within a pedagogically limited perspective of learning as knowledge acquisition (as opposed to construction)” (p. 156). When we began offering our services to academic staff, the majority of academic-conceived video pieces we made were reflective of this paradigm.

We have found that making video lectures (with the lecturer's talking head and supporting PowerPoint slides) is often a relatively poor use of the video medium. The key strength of video is that it (usually) contains both visual and auditory channels, and that these unfold over a period of time, rather than being static snapshots. However, with lengthy straight-to-camera presentations by the teacher, what needs to be learned is communicated largely on the audio channel. There is usually little visual communication or engagement to support the meaning of what is said. The lasting visual impression on the viewer is the single image of the teacher on screen. Image-based PowerPoint slides do improve matters. "Death by PowerPoint” (Kapterev, 2007) is as much a problem in videos as it is in live lectures characterised by reliance on walls of text to match the dialogue and duplicating the modes of communication. This explicit transmission of content disengages students and ignores the strengths of the video format to "show not tell." Teachers also tend to translate their one-hour lectures directly to one-hour videos which is entirely too long for unbroken to-camera delivery, however engaging the teacher is. Finally, if a webcam is used for recording, we have found that academic staff tend to monitor their own images rather than looking into the camera itself (and therefore connect with the audience) which tends to reduce the efficacy of the video (see Figure 1).

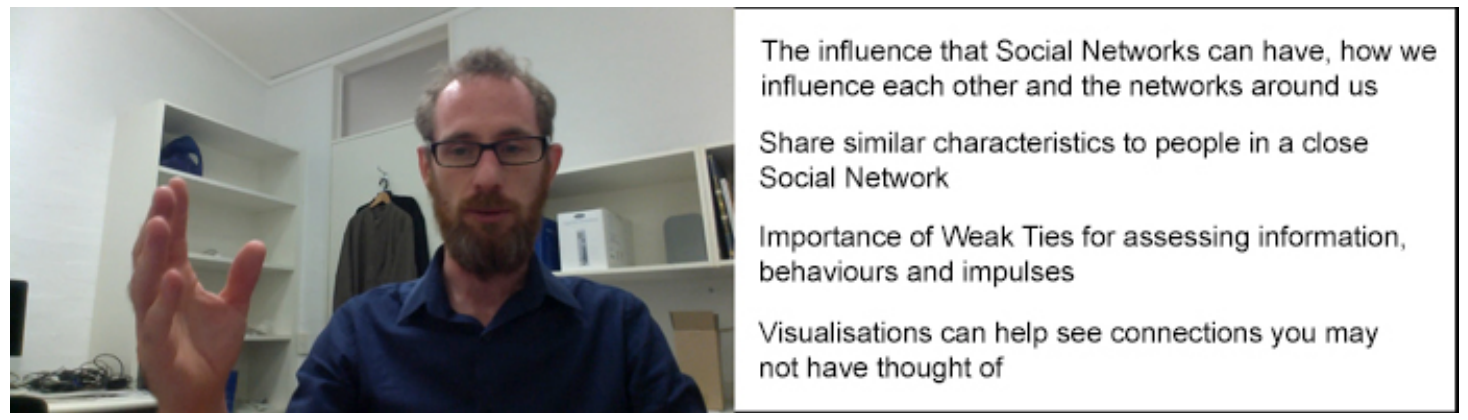

Figure 1. Lecture material recorded on a webcam in the office combined with PowerPoint slides

Even if higher production values are brought to bear, the video lecture may not represent the most effective pedagogical strategy. Here we provide an example - the multi-cam production for a 
traditional lecture (see Figure 2): it follows the model of a standard lecture with PowerPoint slides transposed into the video medium. However, rather than a single camera pointed at the teacher, several cameras were used to capture various angles of the teacher presenting (as per a multi-cam studio television program). Periodically changing the shot-style of each camera, using close-ups, wide shots, and following the teacher around the space added some variety and interest. We found that the multi-cam lecture did improve the level of viewer engagement through a more expansive use of the medium. However, it added little to the learning experience beyond what might have been achieved through a single camera lecture recording with PowerPoint slides. Viewers noted that the only real standout aspect to watching this multi-cam lecture was the camera pointed back at the students from the teacher's perspective ("the reverse shot"), which is not a usual camera position for this type of video. This, however, was deemed to be a superficial way to increase viewer attention that did little to add to the value of the learning. In all, the multi-cam recording of the lecture was a resource-intensive exercise requiring considerable effort for filming, and also in the editing, for an insubstantial gain in learning potential beyond a single-camera filmed lecture.

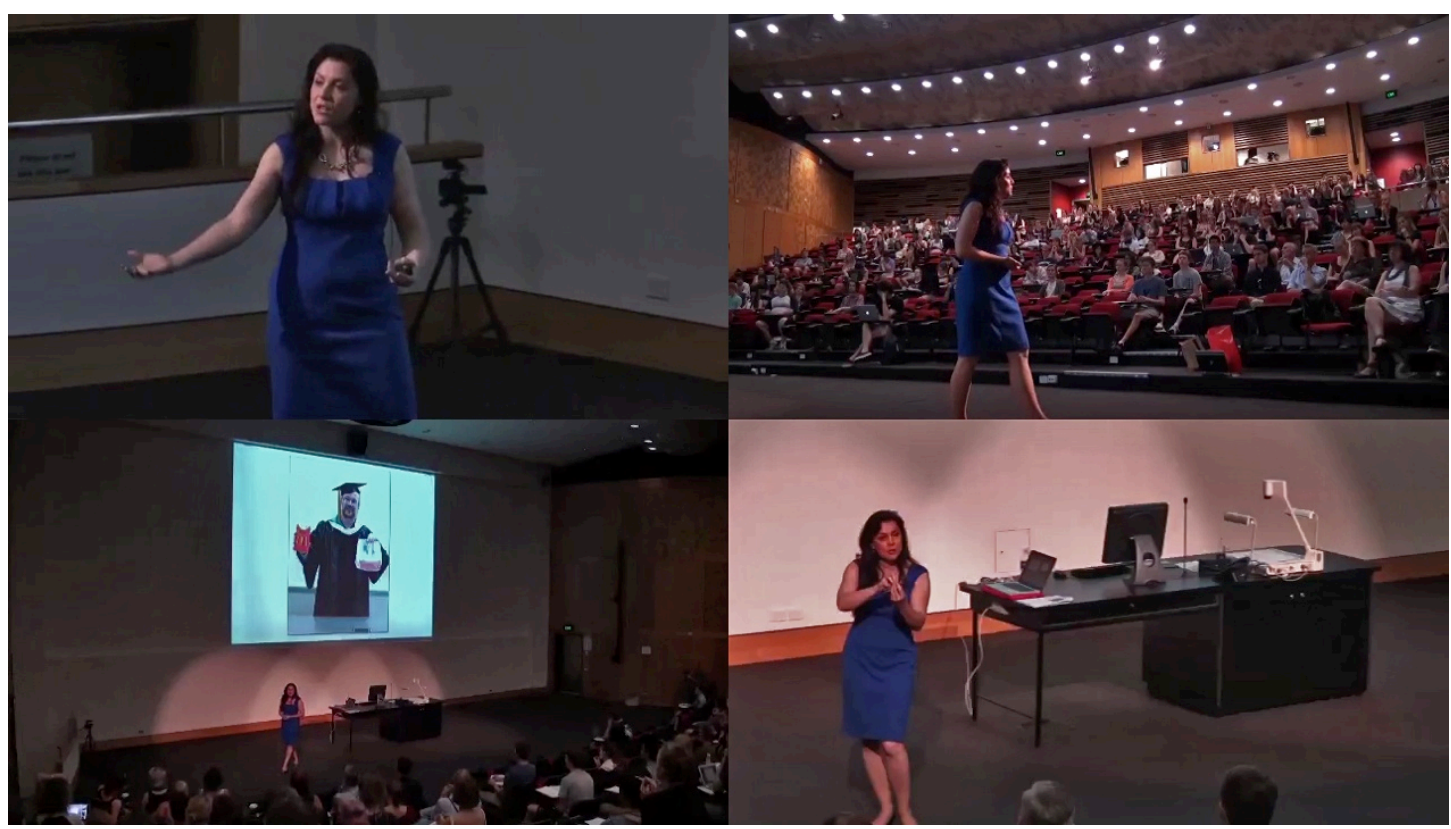

Figure 2. Multi-cam recorded lecture, four cameras with operators, edited together with PowerPoint slides

The video tutorial is arguably a better use of the medium than lectures because it exploits the visual strengths of video. Making use of screen capture software packages such as Camtasia (http://www.techsmith.com/camtasia.html) or Screencast-o-matic (http://www.screencast-omatic.com/), teachers can record actions taking place on a computer screen in real time e.g., demonstrating use of software or stepping students through an online process. The processes and actions appear in the main screen while a smaller image of the presenter's face via the computers webcam can be captured along with the stream. This method is extremely useful for instructional demonstrations. However, without practice or the application of clever techniques outside of these software packages, it can be difficult to compose strong visual engagement with the limitations of what can be manipulated in real time on the computer screen, and combine it with the teacher's voice or talking head. The "split focus" of action and teacher may actually reduce audience engagement and the teacher often appears distracted in the secondary shot as they are focussed on enacting the demonstration rather than connecting with the audience via the camera. In some instances, we have found that it is more effective to remove the secondary shot of the teacher entirely.

Video can, however, lend itself to a student-centred constructivist paradigm rather than a transmissive one through the creation of a problem-solving or enquiry-based learning 
environment. If a range of online and face-to-face learning items are presented and the student is able to build their own learning experience through engaging with the resources in a way that meets an investigatory need, better depth of learning occurs along with the likelihood of curiosity and further engagement with the learning experience (Jonassen, Howland, Moore, \& Marra, 2003).

\section{When should video be used?}

As previously noted, our early efforts in making educational videos with academic staff tended to produce "online lecture" videos wherein the teacher delivered material to camera that would otherwise be delivered in a lecture hall. Thus, the videos often comprised the teacher explaining the entirety of the scheduled learning experience - including administrative elements (announcements, explanations of assessment) as well as all course content - with still PowerPoint slides sometimes interpolated as supporting visual aids. We have found that, irrespective of the overall pedagogic approach taken (e.g., transmissive/behaviourist vs student centred/constructivist), video is much less effective when it comprises the sum total of the standard lecture/learning experience.

While it may be simple and quick to aggregate all of the learning for a week into a video, far better learning results from using a combination of learning resources with each resource matched appropriately to concepts within the content and the intended learning outcomes. This idea of "medium matching the message" means exploiting its specific strengths of video as a visual channel of communication - for instance, as a means to demonstrate skills as per the video tutorial. Non-visual content may be better presented via text or audio, and non-moving images may also be preferred for some content. Using video in instruction and learning should be no more special than using textual resources (Marchionini, 2003).

Koumi (2006) has described three key types of educational value that video is well placed to add: (a) cognitive value, (b) nurturing value, and (c) experiential value. Cognitive value can be added through visual strategies such as demonstrations of processes through animated diagrams, showing "real world" examples or demonstrations. Nurturing value refers to connecting with the audience through the affective domain. Videos can be used to increase motivation to learn through humour, entertainment or inspiration. The experiential value of video relates to vicarious learning, that is, to show aspects of places and interactions between people. It may be easier, less resource-intensive and more effective to take students on a video-based "virtual field trip" to show them situated exemplars rather than to take them to visit a place of interest (Koumi, 2006).

Our work with a Landscape Architect teacher in displaying various aspects of design in a new suburban development around waterflows through a number of short video provides an effective example of matching of message to medium (see Figure 3). The field trip could be hampered by factors such as the weather, the teacher being able to clearly communicate with a large cohort in a public space, travel logistics, insurance concerns and cost. In the lecture, the video segments describing the particular concepts were paired with a map of the overall area to spatially link the separate videos together. The segments, ranging from only 30 seconds to a maximum 2 minutes, then became the basis for further discussion in the classroom where it was easier for the cohort to communicate. 


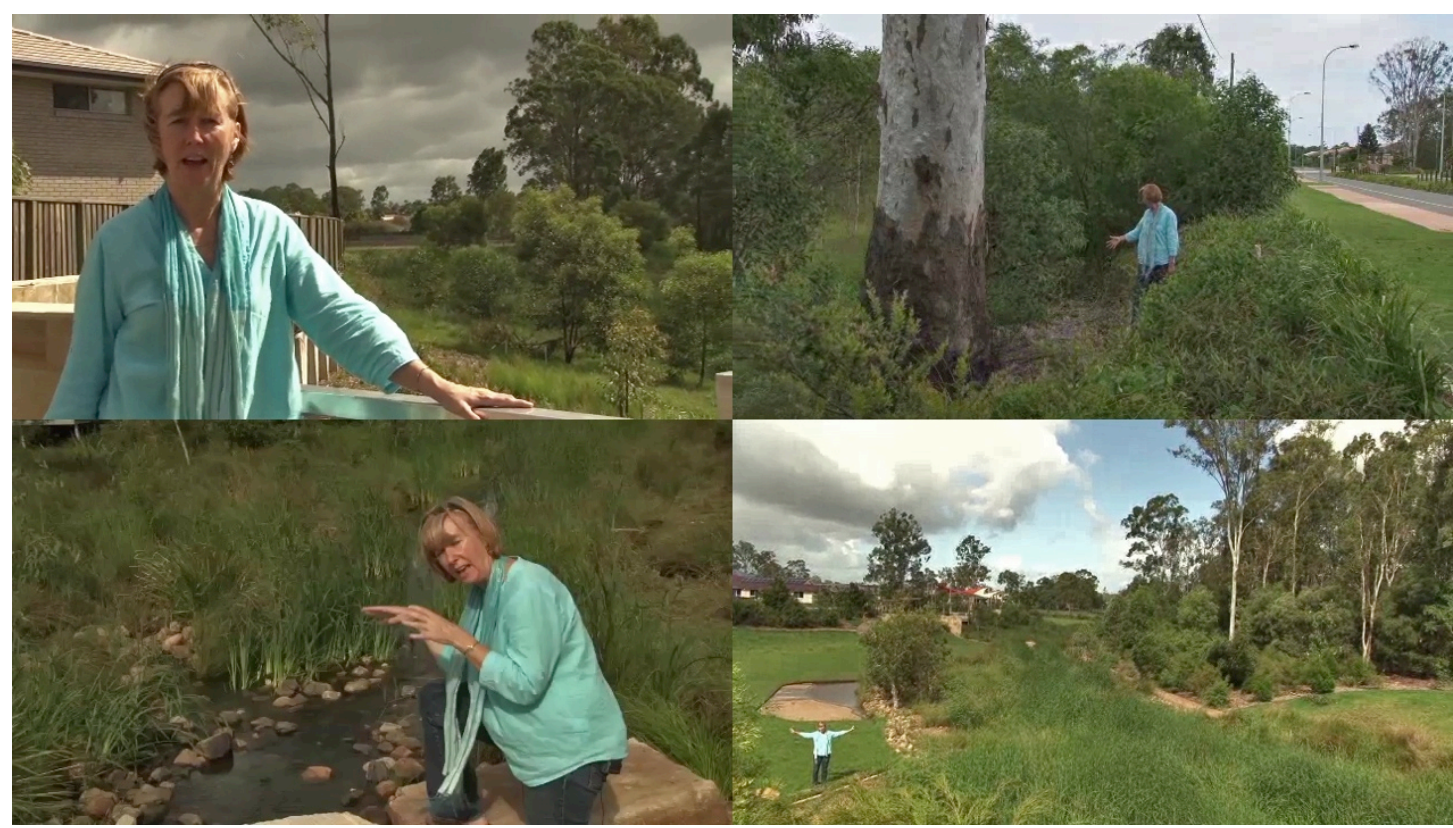

Figure 3. A number of short video segments on location bringing a field trip into to the classroom

Equally, making a video of an interview with a subject matter expert to play later in class may be useful when the interviewee may not be available to attend the class themselves or if more indepth review is needed. In the same way that being able to communicate with family, peers or colleagues is improved through the use of video conferencing, the medium of video enables a much greater depth of engagement with people through seeing them move as well as hearing them, perhaps adding further depth and meaning through filming them in situ (see Figure 4).

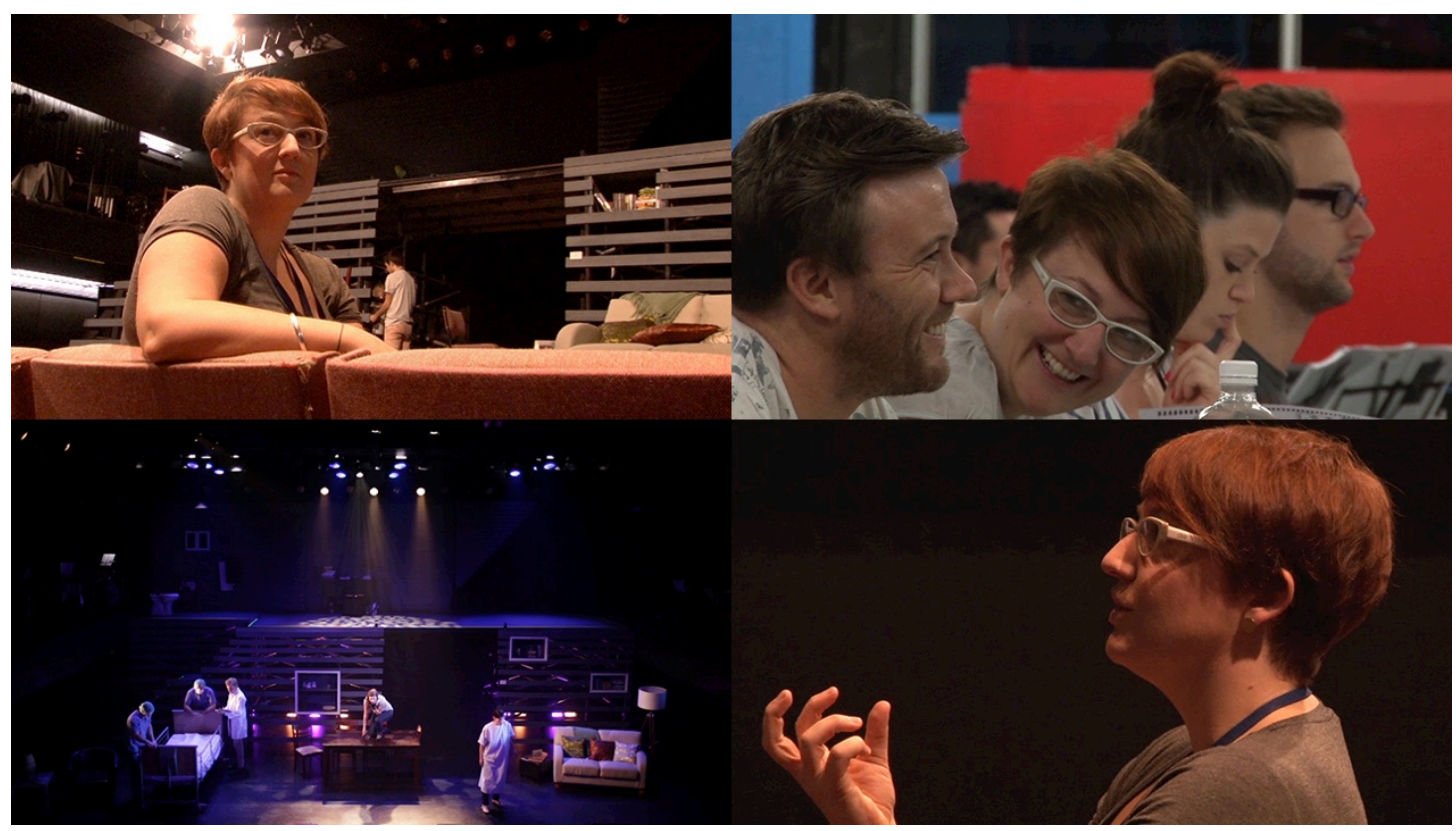

Figure 4. Industry expert interviewed to contextualise and add depth to learning

Depending on the content, it may not be necessary to custom make a video. Many educational possibilities exist in reusing video segments drawn from existing repositories (see Goldberg \& 
LaMagna, 2012 for an overview of found resource repositories). Some of the most prominent education video repositories at present include: Lynda.com (for digital, business and creative skills); Khanacademy.org (maths and science); and Academicearth.org (all subject areas). Youtube.com and Vimeo.com are additional general sources of "free" educational video content. Depending on licensing arrangements, these can be edited for purpose and used in conjunction with custom videos and other types of found and bespoke learning resources. Creative Commons Licensing, which can be applied to YouTube or Vimeo resources, makes this especially exciting as online platforms become more cognisant of and prepared for our remix culture. However, as video is able to be uploaded to these sites by anyone — whether qualified or not - the quality of content may be variable and must therefore be vetted carefully before use.

\section{Making effective videos}

When compared to other educational media possibilities such as print, still images or audio, video (with its unfolding visual and audio channels of communication) is complex and resourceintensive to develop. Invariably we have found that the best outcomes are achieved with rigorous planning through a process of scripting, storyboarding, and pre-assemblage of supporting materials such as still images. We have developed four principles for planning educational videos: (i) Give context and align purpose; (ii) Tell (show) a story; (iii) Keep it as short as possible; and (iv) Present with authenticity.

\section{i. Give context and align purpose}

In accordance with the principles of constructive alignment (Biggs \& Tang, 2007), the content of the video and its intended purpose should be clearly signposted to maximise the likelihood of viewing, and to ensure maximum comprehension and meaning-making through the learning experience. We suggest that this signposting does not need to be in the video itself because the video will exist surrounded by other elements that can serve the purpose, for example, the webpage it is embedded in, the repository details, the hyperlink to it, and even its filename. The reasons for this are twofold:

- $\quad$ the less video-based verbal or written commentary/explanation around the core learning message, the greater the engagement; and also,

- removing “administrative” elements from the video such as explanations of associated assessment items means that the footage may be reusable in other learning contexts and/or for other subjects.

An exception to this might be when explanation of an assessment item would be enhanced through visual support. For instance, a video describing assessment tasks we helped to develop for an Architecture unit on Visualisation techniques was much improved over textual content with images (see Figure 5). The video contained a large number of past student examples in just a few minutes with an audio description. A webpage containing the same number of photo examples and text would have been large and unwieldy, and so long that students may not bother to read it in its entirety. 


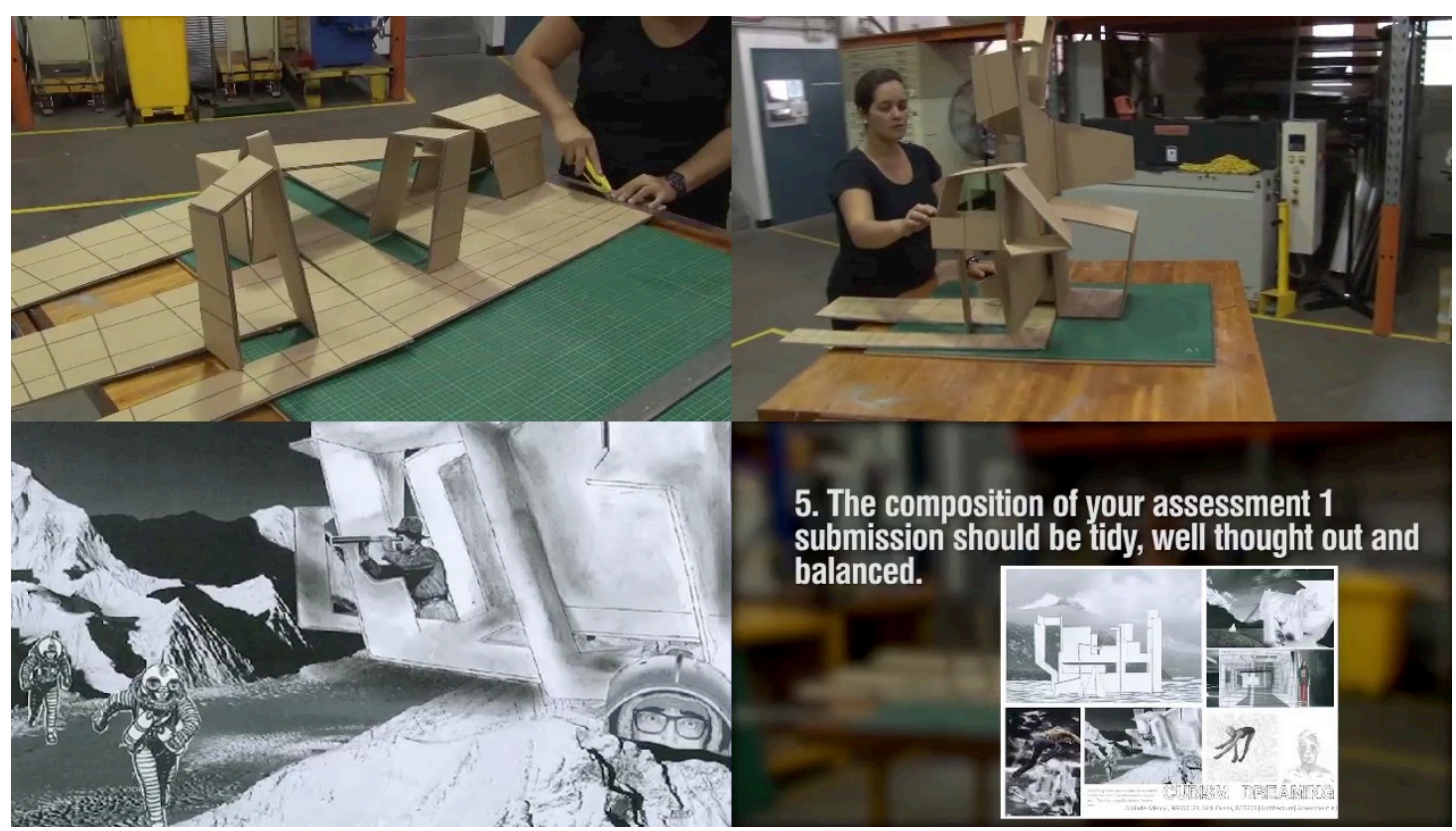

Figure 5. Assessment video with physical demonstration and past student examples

\section{ii. Tell (show!) a story}

In video development, we work with the teachers to construct elements of interest that speak to the aspects of visual storytelling. This often starts with teasing out the teachers' own narratives, experiences and stories that are relevant to the content as well as identifying appropriate locations, contexts and visual aids to go with them. In filming, we apply cinematic principles as far as is possible with minimal equipment (e.g., available lighting, one camera, and sometimes handheld only) and use multiple locations. Relevant, well-composed and complementary overlays of visual material provide an additional channel of communication to maximise retention of concepts. Text on screen is minimised apart from occasional key “cueing concepts” words as direct redundancy on visual and auditory channels is annoying to the viewer and actually reduces retention (Koumi, 2006). These visual strategies are designed to elaborate concepts and pique viewer interest.

\section{iii. $\quad$ Present with authenticity}

It is very rare indeed for anybody to spontaneously demonstrate a natural affinity for presenting to camera without some kind of specific experience, professional development and/or coaching. Presenting to camera is a skill which, like any other, needs to be learned, developed and refined and, in so doing, develops proficiency, presence and confidence. Whilst we are not referring to "performance" - such as that of an actor — presenting to camera does require a measure of selfawareness, physical consciousness and a degree of performance-related imperatives.

Supplementing these self-awareness skills, on-camera confidence and presence are equally derived from the following:

1. being conversant with the basics of on-camera environment and terminology;

2. detailed, in-depth, fluent knowledge of one's materials;

3. appropriate adaptation and repackaging of those materials for the on-camera context;

4. detailed preparation and rehearsal; and,

5. fundamental belief in oneself to not only speak to the camera lens but indeed to project one's personality through that inanimate, intimidating, unresponsive piece-of-glass.

Confidence and professional presence needs to emerge from the screen and be perceived and believed by the audience. Confidence, academic credibility, and personal authenticity, were thus central motives and motivators for our engagement with the academics' professional development. 
Teachers were initially prepared for the task of presenting to camera through small group workshops designed to bolster their confidence and visual literacy. We have made it a priority that the teachers present in their videos. In doing so, we involve them directly in the space through walking and talking, physically embedding them into and making contact with their environment, and reflecting on the concepts from their own experience through storytelling; thus engaging the viewer through their own passion. In order to maximise engagement, it must seem like the teacher is speaking to an individual student personally and directly — almost like a one on one conversation - even though the teacher knows that the video's purpose is to communicate to the whole cohort.

\section{Iv Keep it short and to the point}

There are no hard and fast rules with respect to exact video lengths. It seems, however, that the way viewers engage with online educational video tends to be different from television or cinema watching. The optimal video length for mobile devices may be less than five minutes as opposed to greater than one hour for television and greater than two hours for cinema (Cann, 2007). This may well be a function of audience expectations relating to the video content and how they tend to interact with various media platforms. Cann (2007) suggested that television and cinema audiences are often in passive "entertain me" mode whereas online viewers of video are more active, with mouse or trackpad in hand ready to click. The average Youtube video length is 4 minutes and 12 seconds and $40 \%$ of the videos are watched on mobile devices (YouTube Statistics, 2013). Because viewer abandonment rates are also far higher for online video than other media, getting to the point quickly is crucial. Therefore, the most important concepts should be presented first if possible. Where there is need to convey a number of concepts, we try to structure that content around a number of shorter videos rather than one longer video. This serves to make the audience feel that their time is not being wasted as well as allowing for some potential for self-directedness in learning by giving the students options around what to watch and when.

This final example of an introduction video for a Typography course exemplifies a number of aspects to our approach (see Figure 6). The script initially provided by the teacher was a truncation of their original lecture. It was certainly brief enough to be contained within a few minutes of video and outlined what the unit was (including administrative details), introduced what the students would be learning, and provided an example of typography that emphasised the learning.

We determined that a much more interesting and engaging video could be constructed by swapping around the sections outlined in the script. It resulted in opening with the example of typography applied in a real world scenario, how that linked with what the students would learn, and concluding with introducing the teacher and how the students could obtain further information. Whilst there was a large degree of transmissive and explicit content in this video's structure, it was however engaging for the audience - they were first asked a question, shown some interesting ideas with good accompanying explanatory visuals, and did not have to wade through the explicit transmission to have their curiosity provoked. 


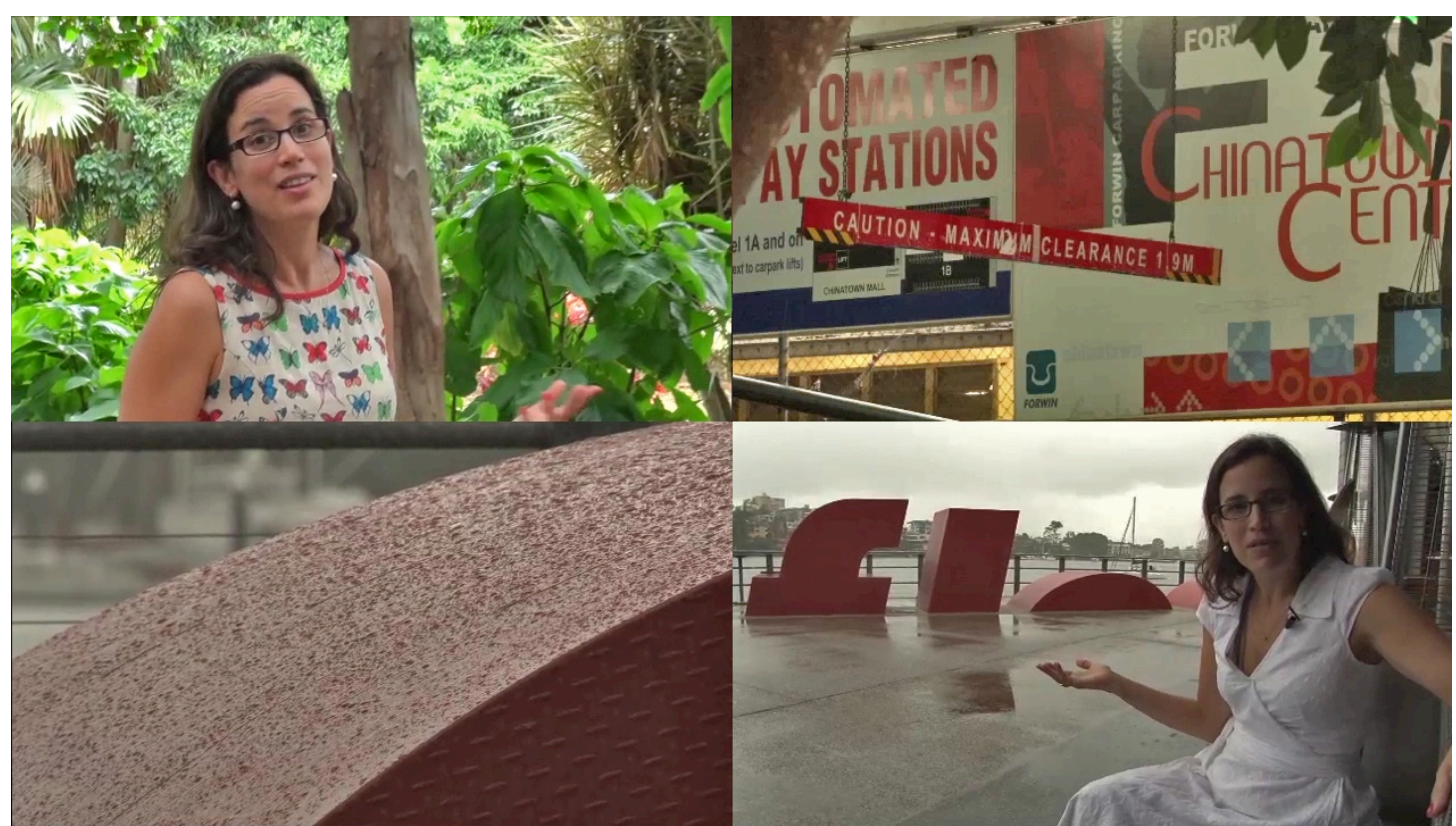

Figure 6. Unit introduction video presenting real world applications of typography

\section{Conclusions}

In this paper, we have shared some of our experience around developing video content for teaching in universities. While video is well established in the entertainment realm, it has only become a vehicle for online and mobile learning relatively recently. Although developing effective content requires significant expertise, the technical requirements have become more accessible through reduced cost and improved technology, thus allowing teachers to make their own content as never before (Marchionini, 2003). Significant further investigations need to be undertaken to enhance knowledge around optimising video as a learning tool, particularly addressing how video can best be used within a suite of learning resources and how interactivity and learner engagement with video can be maximised.

If universities are serious about 21st century pedagogy, they must also provide effective professional learning opportunities and other forms of organisational support for teachers. While creating video is far easier than it has been in the past and the enormous educational opportunities afforded by video and other forms of rich media has been acknowledged, simply providing infrastructure and tools, or training that is technology-focussed ("how to use the widget") (Younie \& Leask, 2013) will likely fail. Professional learning for teachers that is focussed on video must cover technological, pedagogical and curricular domains and is more likely to succeed if the program offers the opportunity to share and collaborate with other teachers, academic developers, and technology experts, and is situated in the context of teachers' ongoing work (Hogue, 2013). In order to encourage experimentation in teaching and transformation of pedagogical practice, university leaders also need to offer positive, supportive and well-aligned success measures, while also supporting academic self-directedness and autonomy. Teachers need to be able to connect their own goals and visions with institutional aims, and at the same time know that experimentation is not only permitted but also encouraged. Making and using videos for teaching can be enormous fun and it is through play that much of the best learning occurs.

\section{References}

Biggs, J., \& Tang, C. (2007). Teaching for quality learning at university (3rd ed.). Maidenhead, UK: Open University Press. 
Bishop, J. L., \& Verleger, M. A. (2013, June). The flipped classroom: A survey of the research. Paper presented at ASEE National Conference Proceedings, Atlanta, GA. Retrieved from http://www.studiesuccesho.nl/wp-content/uploads/2014/04/flippedclassroom-artikel.pdf

Cann, A. J. (2007). Podcasting is dead. Long live video! Bioscience Education, 10. Retrieved from http://www.bioscience.heacademy.ac.uk/journal/vol10/beej-10-c1.aspx. doi: 10.3108/beej.10.c1

Demetriadis, S., \& Pombortsis, A. (2007). E-lectures for flexible learning: A study on their learning efficiency. Educational Technology \& Society, 10(2), 147-157.

Goldberg, E. J., \& LaMagna, M. (2012). Open educational resources in higher education: a guide to online resources. College \& Research Libraries News, 73(6), 334-337.

Hakkarainen, P., Saarelainen, T., \& Ruokamo, H. (2007). Towards meaningful learning through digital video supported, case based teaching. Australasian Journal of Educational Technology, 23(1), 87-109. Retrieved from http://www.ascilite.org.au/ajet/ajet23/hakkarainen.html

Hogue, R. J. (2013). Considerations for a professional development program to support iPads in higher education teaching. Ubiquitous Learning, 5(1), 25-35.

Jonassen, D. H., Howland, J., Moore, J., \& Marra, R. M. (2002). Learning to solve problems with technology: A constructivist perspective (2nd ed.). Upper Saddle River, NJ: Merrill/Prentice Hall.

Kapterev, A. (2007). Death by PowerPoint (and how to fight it). Retrieved from http://www.slideshare.net/thecroaker/death-by-powerpoint

Keegan, H. (2010). Immersed in the digital: Networked creativity through mobile video production. Paper presented at Association for Learning Technology conference. Nottingham, UK, September 2010. Retrieved from http://repository.alt.ac.uk/832

Kiili, C., Kauppinen, M., \& Laurinen, L. (2013). University students as composers of a digital video. Paper presented at Open and Social Technologies for Networked Learning (OST 12). July 30-August 3, 2012. Tallinn University, Estonia.

Koumi, J. (2006). Designing video and multimedia for open and flexible learning. Oxford, UK: Routledge Falmer.

Marchionini, G. (2003). Video and learning redux: New capabilities for practical use. Educational Technology, 43(2), 36-41.

Mateer, G. (2011). Using media to enhance teaching and learning. Starting Point: Teaching and Learning Economics. Retrieved from http://serc.carleton.edu/econ/media/index.html

Younie, S, \& Leask, M. (2013). Teachers, pedagogies, and professional development. Teaching with Technologies: The Essential Guide (pp. 83-109). Maidenhead, Berkshire, UK.: Open University Press.

YouTube Statistics. (2013). Retrieved from http://www.youtube.com/yt/press/statistics.html 


\section{Acknowledgments}

The authors would like to thank the QUT CIF RE-Delivery initiative participants, and acknowledge particularly the presenters in the video screenshots used in this paper: Gillian Lawson, Emily Gilhome, Manuela Taboada and Glenda Caldwell.

Copyright @ 2014 Andrew Thomson, Ruth Bridgstock and Christiaan Willems 